\title{
EMG Activation Patterns Associated with High Frequency, Long-Duration Intracortical Microstimulation of Primary Motor Cortex
}

\author{
Darcy M. Griffin, Heather M. Hudson, Abderraouf Belhaj-Saï, and Paul D. Cheney \\ Department of Molecular and Integrative Physiology, University of Kansas Medical Center, Kansas City, Kansas 66160
}

The delivery of high-frequency, long-duration intracortical microstimulation (HFLD-ICMS) to primary motor cortex (M1) in primates produces hand movements to a common final end-point regardless of the starting hand position (Graziano et al., 2002). We have confirmed this general conclusion. We further investigated the extent to which the (1) temporal pattern, (2) magnitude, and (3) latency of electromyographic (EMG) activation associated with HFLD-ICMS-evoked movements are dependent on task conditions, including limb posture. HFLD-ICMS was applied to layer V sites in M1 cortex. EMG activation with HFLD-ICMS was evaluated while two male rhesus macaques performed a number of tasks in which the starting position of the hand could be varied throughout the workspace. HFLDICMS-evoked EMG activity was largely stable across all parameters tested independent of starting hand position. The most common temporal pattern of HFLD-ICMS-evoked EMG activity ( $58 \%$ of responses) was a sharp rise to a plateau. The plateau level was maintained essentially constant for the entire duration of the stimulus train. The plateau pattern is qualitatively different from the largely bell-shaped patterns typical of EMG activity associated with natural goal directed movements (Brown and Cooke, 1990; Hoffman and Strick, 1999). HFLD-ICMS produces relatively fixed parameters of muscle activation independent of limb position. We conclude that joint movement associated with HFLD-ICMS occurs as a function of the length-tension properties of stimulus-activated muscles until an equilibrium between agonist and antagonist muscle force is achieved.

Key words: corticospinal; electromyography; ICMS; intracortical microstimulation; motor cortex; motor mapping

\section{Introduction}

Intracortical microstimulation (ICMS) is a method with relatively high spatial resolution used to study the organization and function of brain motor areas (Stoney et al., 1968). Characterizing movements evoked with high-frequency, long-duration (500 ms) ICMS (HFLD-ICMS) has provided interesting new insight into the actions of ICMS on motor circuits (Graziano et al., 2002; Aflalo and Graziano, 2006a, b). One result derived from application of this method was the demonstration that stimulation of individual sites in frontal cortex drives the hand to a consistent final end-point position regardless of the starting position of the

Received Aug. 26, 2013; revised Nov. 27, 2013; accepted Dec. 15, 2013.

Author contributions: D.M.G., H.M.H., A.B.-S., and P.D.C. designed research; D.M.G., H.M.H., A.B.-S., and P.D.C performed research; D.M.G., H.M.H., and P.D.C. analyzed data; D.M.G., H.M.H., and P.D.C. wrote the paper.

This work was supported by NIH Grants NS051825 and NS064054, NIH Center Grant HD02528, the Kathleen M. Osborn Endowment (P.D.C.), and the KUMC Biomedical Research Training Program (D.M.G.). We thank lan Edwards for technical assistance.

Correspondence should be addressed to Dr. Paul D. Cheney, University of Kansas Medical Center, Department of Molecular and Integrative Physiology, 3901 Rainbow Boulevard, Mailstop 3043, Kansas City, KS 66160-7336. E-mail: pcheney@kumc.edu.

D. Griffin's present address: Systems Neuroscience Institute, University of Pittsburgh School of Medicine, Pittsburgh, PA, 15261.

H. Hudson's present address: Department of Neurology, University of Minnesota School of Medicine, Minneapolis, MN, 55455.

DOI:10.1523/JNEUROSCI.3643-13.2014

Copyright $\odot 2014$ the authors $\quad 0270-6474 / 14 / 341647-10 \$ 15.00 / 0$ hand or posture of the arm. The mechanism responsible for HFLD-ICMS-evoked movements has not been clarified.

One hypothesis that might explain the results of HFLD-ICMS is that the stimulus activates a natural physiological output circuit which is modified by afferent feedback to make it appropriate for the direction of movement needed to achieve a target position relative to the current position of the hand (Graziano et al., 2004; Aflalo and Graziano, 2006a). The natural circuit hypothesis predicts that HFLD-ICMS-evoked EMG responses will be highly dependent on arm posture and joint angle. Thus, to achieve the same end-point position of the hand, HFLD-ICMS should produce activation of flexors and suppression of extensors at a joint for one starting arm posture but the reverse muscle activation pattern for another starting posture where the joint must move in the opposite direction to achieve the same final target position (Graziano et al., 2002). The natural circuit hypothesis predicts that HFLD-ICMS-evoked muscle activation will resemble the pattern of activation associated with natural voluntary movement.

An alternative hypothesis is that the stimulus hijacks the motor circuitry replacing natural voluntary activity with activity that is largely stimulus driven (Griffin et al., 2011). In this case, the descending stimulus-driven activity dominates motoneuron activity. Joint movement occurs as a function of the length-tension properties of the activated agonist and antagonist muscles and continues until a new equilibrium position is achieved. We refer 
to this as stimulus-driven equilibrium. The neural hijacking hypothesis (Griffin et al., 2011) predicts that HFLD-ICMSevoked responses will be relatively independent of initial arm posture and joint angle. The objective of this study was to test these hypotheses concerning the mechanism of HFLD-ICMS-evoked movements by investigating the underlying characteristics of muscle activation.

We have quantified the extent to which the sign (facilitation or suppression), magnitude, and temporal pattern of HFLDICMS-evoked EMG activity varies as a function of arm posture. We have found that HFLD-ICMS produces relatively fixed parameters of muscle activation, independent of starting hand position. In the majority of cases, the pattern of activity is tonic with a rise to a constant level, which is largely maintained throughout stimulation. Our results support the hijacking hypothesis and also suggest that the final common end-point position, characteristic of HFLD-ICMS-evoked movements, results from movement toward an equilibrium position along the length-tension curves of the stimulus-activated agonist and antagonist muscles at each joint.

\section{Materials and Methods}

Behavioral tasks. HFLD-ICMS (100 biphasic stimulus pulses at $200 \mathrm{~Hz}, 500$ ms train duration, 60 and $120 \mu$ A stimulus intensities) was applied to the left primary motor (M1) cortex of two male rhesus monkeys (Macaca mulatta; $\sim 10 \mathrm{~kg}$, 9 years old) while they reached with their right hand for a food reward (Fig. 1Aa) or while they performed an isolated wrist task (Fig. 1B). HFLD-ICMS was also applied to the left M1 cortex of one male rhesus monkey (Monkey A) while he reached with his right hand to a handle placed in various positions within the workspace (Fig. $1 \mathrm{Aa}$ ). The wrist task was performed either concentrically with step movements between flexion and extension position zones or isometrically with the manipulandum locked in place at two different wrist positions. During each data collection session, the monkey was seated in a custom-built primate chair inside a soundattenuating booth. The left arm was restrained during task performance. All tasks were performed with the right arm/hand.

Hand starting positions for the reaching task are illustrated in Figure $1 \mathrm{Aa}$. Both monkeys were offered peanuts in various positions around the work space (Fig. $1 A a$, yellow circles and numbers). HFLD-ICMS was delivered as the monkey's hand entered the target location, but before the monkey grasped the reward. Alternatively, one monkey (Monkey A) was required to grip a handle that was locked into place at up to four different positions within the monkey's work space (Fig. $1 A a$, blue circles and letters). The handle was coupled to a force transducer and the monkey was required to produce force in either the push or pull direction. HFLDICMS was delivered during push and pull force generation at all of the four positions in the workspace.

Shoulder and elbow angles associated with starting hand positions of the reaching tasks are listed in Table 1. Joint angles were measured using photographs of the monkey's arm at each of the starting hand positions. Digital images were processed in ImageJ using the shoulder, ribcage, elbow, and wrist joints as base points on the body. Final angle measurements are an average from several sessions. Figure $1 A$ illustrates how the shoulder and elbow measurements were made in both the vertical (Fig. $1 A b)$ and horizontal (Fig. $1 A c$ ) planes.

For the wrist task (Fig. 1B), the monkey's lower and upper arm were restrained. The hand, with digits extended, was placed in a padded ma-
Table 1. Joint angles achieved with different hand starting positions

\begin{tabular}{|c|c|c|}
\hline Hand starting positions & Joint & Angle \\
\hline \multirow[t]{2}{*}{ A } & Shoulder & $\begin{array}{l}50^{\circ} \text { Horizontal plane } \\
15^{\circ} \text { Vertical plane }\end{array}$ \\
\hline & Elbow & $105^{\circ}$ Horizontal plane \\
\hline \multirow[t]{2}{*}{ B } & Shoulder & $\begin{array}{l}50^{\circ} \text { Horizontal plane } \\
125^{\circ} \text { Vertical plane }\end{array}$ \\
\hline & Elbow & $115^{\circ}$ Horizontal plane \\
\hline \multirow[t]{2}{*}{ C } & Shoulder & $\begin{array}{l}110^{\circ} \text { Horizontal plane } \\
125^{\circ} \text { Vertical plane }\end{array}$ \\
\hline & Elbow & $135^{\circ}$ Vertical plane \\
\hline \multirow[t]{2}{*}{ D } & Shoulder & $\begin{array}{l}110^{\circ} \text { Horizontal plane } \\
115^{\circ} \text { Vertical plane }\end{array}$ \\
\hline & Elbow & $120^{\circ}$ Vertical plane \\
\hline \multirow[t]{2}{*}{1} & Shoulder & $\begin{array}{l}50^{\circ} \text { Horizontal plane } \\
120^{\circ} \text { Vertical plane }\end{array}$ \\
\hline & Elbow & $70^{\circ}$ Vertical plane \\
\hline \multirow[t]{2}{*}{2} & Shoulder & $\begin{array}{l}110^{\circ} \text { Horizontal plane } \\
120^{\circ} \text { Vertical plane }\end{array}$ \\
\hline & Elbow & $70^{\circ}$ Vertical plane \\
\hline \multirow[t]{2}{*}{3} & Shoulder & $\begin{array}{l}90^{\circ} \text { Horizontal plane } \\
120^{\circ} \text { Vertical plane }\end{array}$ \\
\hline & Elbow & $170^{\circ}$ Vertical plane \\
\hline \multirow[t]{2}{*}{4} & Shoulder & $\begin{array}{l}90^{\circ} \text { Horizontal plane } \\
120^{\circ} \text { Vertical plane }\end{array}$ \\
\hline & Elbow & $90^{\circ}$ Vertical plane \\
\hline \multirow[t]{2}{*}{5} & Shoulder & $\begin{array}{l}90^{\circ} \text { Horizontal plane } \\
90^{\circ} \text { Vertical plane }\end{array}$ \\
\hline & Elbow & $90^{\circ}$ Vertical plane \\
\hline
\end{tabular}

Angles estimated to the nearest $5^{\circ}$. See Figure 1 for identification of starting hand positions.

nipulandum that rotated about the wrist. The wrist was aligned with the axis of rotation of the torque wheel to which the manipulandum was attached. The monkey was required to make wrist movements alternating between flexion and extension position zones. Both position zones had an inner boundary of $20^{\circ}$ and an outer boundary of $40^{\circ}$. HFLDICMS was delivered at the beginning of the target hold period. Alternatively, the monkey performed an isometric wrist task with the manipulandum locked in place at $30^{\circ}$ of flexion or $30^{\circ}$ of extension. In 
this case, the monkey was required to generate ramp and hold wrist torque trajectories alternately between flexion and extension target zones. The inner and outer boundaries of the torque window were 0.025 $\mathrm{N} \cdot \mathrm{m}$ and $0.05 \mathrm{~N} \cdot \mathrm{m}$, respectively, for flexion and $0.008 \mathrm{~N} \cdot \mathrm{m}$ and 0.025 $\mathrm{N} \cdot \mathrm{m}$, respectively, for extension. HFLD-ICMS was delivered at the beginning of the target hold period. HFLD-ICMS was only applied once every 3-4 trials to minimize disruption of task performance and to ensure successful completion of a sufficient number of trials to maintain the monkey's interest.

Each HFLD-ICMS experiment was recorded using a hand held Sony digital video tape recorder. Stimulus driven hand end-points were determined at the end of each recording session and verified by video playback.

Surgical procedures. After training, a $30 \mathrm{~mm}$ inside diameter titanium chamber was stereotaxically centered over the left M1 forelimb area of each monkey and anchored to the skull with 12 titanium screws (Stryker) and dental acrylic (Lux-it). Threaded titanium nuts (Titanium Unlimited) were attached over the occipital aspect of the skull using 12 additional titanium screws and dental acrylic. These nuts provided a point of attachment for a flexible head restraint system for use during data collection sessions. The chambers were centered at anterior $16 \mathrm{~mm}$, lateral 18 $\mathrm{mm}$ (Monkey V) and anterior $16 \mathrm{~mm}$, lateral $22 \mathrm{~mm}$ (Monkey A), at a $30^{\circ}$ angle to the midsagittal plane.

EMG activity was recorded from 24 contralateral (right) forelimb muscles with pairs of insulated, multistranded stainless steel wires (Cooner Wire, AS632) implanted during aseptic surgical procedures (Park et al., 2000). Pairs of wires for each muscle were tunneled subcutaneously from an opening above the elbow to their target muscles. The wires of each pair were bared of insulation for $\sim 2-3 \mathrm{~mm}$ at the tip and inserted into the muscle belly with a separation of $\sim 5 \mathrm{~mm}$. Implant locations were confirmed by stimulation through the wire pair and observation of appropriate muscle twitches and joint movements. EMG connector terminals (ITT Cannon) were affixed to the upper arm using medical adhesive tape. Following surgery, the monkeys wore Kevlar jackets (Lomir Biomedical) reinforced with fine stainless steel mesh (Sperian Protection Americas) to protect the implants. EMG activity was recorded from five shoulder muscles: pectoralis major (PEC), anterior deltoid (ADE), posterior deltoid (PDE), teres major (TMAJ) and latissimus dorsi (LAT); seven elbow muscles: biceps short head (BIS), biceps long head (BIL), brachialis (BRA), brachioradialis (BR), triceps long head (TLON), triceps lateral head (TLAT), and dorso-epitrochlearis (DE); five wrist muscles: extensor carpi radialis (ECR), extensor carpi ulnaris (ECU), flexor carpi radialis (FCR), flexor carpi ulnaris (FCU), and palmaris longus (PL); five digit muscles: extensor digitorum communis (EDC), extensor digitorum 2,3 (ED 2,3), extensor digitorum 4,5 (ED 4,5), flexor digitorum superficialis (FDS), and flexor digitorum profundus (FDP); and two intrinsic hand muscles: abductor pollicis brevis (APB) and first dorsal interosseus (FDI).

All surgeries were performed under deep general anesthesia and aseptic conditions. Postoperatively, monkeys were given an analgesic (buprenorphine $0.5 \mathrm{mg} / \mathrm{kg}$ every $12 \mathrm{~h}$ for $3-4 \mathrm{~d}$ ) and antibiotics (penicillin $\mathrm{G}$, benzathaine/procaine combination, $40,000 \mathrm{IU} / \mathrm{kg}$ on the day before surgery and 3 days later.). All procedures were approved by the Institutional Animal Care and Use Committee and were in accordance with the Association for Assessment and Accreditation of Laboratory Animal Care and the Guide for the Care and Use of Laboratory Animals, published by the US Department of Health and Human Services and the National Institutes of Health.

Data collection and analysis. Sites in M1 were stimulated using glass and Mylar-insulated platinum-iridium electrodes with impedances ranging from 0.5 to $1.2 \mathrm{M} \Omega$ (Frederick Haer). The electrode was positioned within the chamber using an $x-y$-coordinate manipulator and was advanced approximately at a right angle into the cortex with a manual hydraulic microdrive (Frederick Haer). Rigid support for the electrode was provided by a 22 gauge guide tube (Small Parts) inside a $25 \mathrm{~mm}$ long, $3 \mathrm{~mm}$ diameter stainless steel post which served to guide the electrode to the dural surface.

First cortical unit activity was noted and the electrode was lowered 1.5 $\mathrm{mm}$ below this point to layer V. Several steps were taken to distinguish layer $\mathrm{V}$ from more superficial layers, particularly in the bank of the precentral gyrus. First, neuronal activity was evaluated for the presence of large action potentials that were often modulated with the task. Second, effects in stimulus-triggered averages (StTAs) were evaluated for the presence of robust effects at low stimulus intensities $(15 \mu \mathrm{A})$. Individual stimuli were symmetrical biphasic pulses: a $0.2 \mathrm{~ms}$ negative pulse followed by a $0.2 \mathrm{~ms}$ positive pulse. Stimulus pulses were generated and their timing was recorded concurrently with task signals and EMG activity. EMG activity was generally filtered from 30 to $1 \mathrm{kHz}$, digitized at a rate of $4 \mathrm{kHz}$ and full-wave rectified.

Stimulus triggered averages. Stimuli (individual biphasic stimulus pulses at $15 \mathrm{~Hz}, 15$ and $30 \mu \mathrm{A}$ stimulus intensities) were applied to layer $\mathrm{V}$ sites throughout all phases of the tasks and were used to construct StTAs of rectified EMG activity. The assessment of StTA effects was based on averages of at least 500 trigger events. StTAs were compiled over a 60 $\mathrm{ms}$ epoch, including $20 \mathrm{~ms}$ before the trigger to $40 \mathrm{~ms}$ after the trigger. At each stimulation site, averages were obtained for all 24 muscles. Mean baseline activity and SD of baseline EMG activity was measured from the pretrigger period typically consisting of the first $12.5 \mathrm{~ms}$ of each average. StTAs were considered to have significant poststimulus facilitation (PStF) if the points of the record crossed a level equivalent to $2 \mathrm{SD}$ of the mean of the baseline EMG for a period $\geq 0.75 \mathrm{~ms}$ ( 3 points) or more (Park et al., 2001). Note that an effect with a minimum width of $0.75 \mathrm{~ms}$ at the peak would typically have a much longer duration at the baseline in the range of 3-4 ms.

EMG segments associated with each stimulus were accepted as valid only if the mean of all EMG data points over the entire $60 \mathrm{~ms}$ epoch was $\geq 5 \%$ of full-scale signal input. This criterion prevented averaging segments in which EMG activity might be absent. Because the method detects changes in the firing probability of motor units, EMG segments lacking activity are invalid (McKiernan et al., 1998). EMG recordings were tested for cross talk by computing EMG-triggered averages (Cheney and Fetz, 1980). This procedure involved using the EMG peaks from one muscle as triggers for compiling averages of rectified EMG activity from that muscle and all other muscles. Most muscles showed no evidence of crosstalk. However, in muscles that did have crosstalk peaks, we still accepted the effects as valid if the ratio of PStF between the test and trigger muscles exceeded the ratio of their crosstalk peaks by a factor of two or more (Buys et al., 1986). Based on this criterion, none of the effects obtained in this study were eliminated.

HFLD-ICMS-evoked EMG activity. Layer V sites, defined as having one or more clear PStF effects in $15 \mu \mathrm{A}$ StTAs of recorded forelimb muscles, were identified and selected for data collection with HFLD-ICMS. HFLD-ICMS consisted of a train of 100 symmetrical biphasic stimulus pulses $(200 \mathrm{~Hz}, 500 \mathrm{~ms}$ train duration) at stimulus intensities of 60 and $120 \mu \mathrm{A}$. The assessment of HFLD-ICMS elicited effects was based on averages of 4-10 trigger events. The first pulse of each train was used as a trigger to compute averages of HFLD-ICMS-evoked EMG activity.

HFLD-ICMS-evoked EMG activity was compiled over a $1200 \mathrm{~ms}$ epoch, including $200 \mathrm{~ms}$ before the trigger to $1000 \mathrm{~ms}$ after the trigger. At each stimulation site, averages were obtained for all 24 muscles. Mean baseline activity was measured from the pretrigger period typically consisting of the first $100 \mathrm{~ms}$ of each average. HFLD-ICMS-evoked muscle activity was evaluated for (1) the sign of activation (increase or decrease relative to the baseline), (2) mean level of activity during stimulation, (3) temporal pattern, and (4) onset latency. The onset latency of the HFLDICMS-evoked EMG activity was clear based on visual inspection and was identified as the point where the record left the pretrigger baseline toward a peak or trough. The magnitude of the EMG response was expressed as the mean EMG level associated with the $500 \mathrm{~ms}$ stimulus train.

Imaging. Structural magnetic resonance images (MRIs) were obtained from a 3 Tesla Siemens Allegra system. Images were obtained with the monkey's head mounted in an MRI compatible stereotaxic apparatus so the orientation and location of the cortical recording chamber and electrode track penetrations could be determined. A two-dimensional rendering of experimental sites was constructed for each monkey. The locations of experimental sites were mapped onto a two-dimensional cortical sheet based on the electrode's depth and $x$-y-coordinate. 


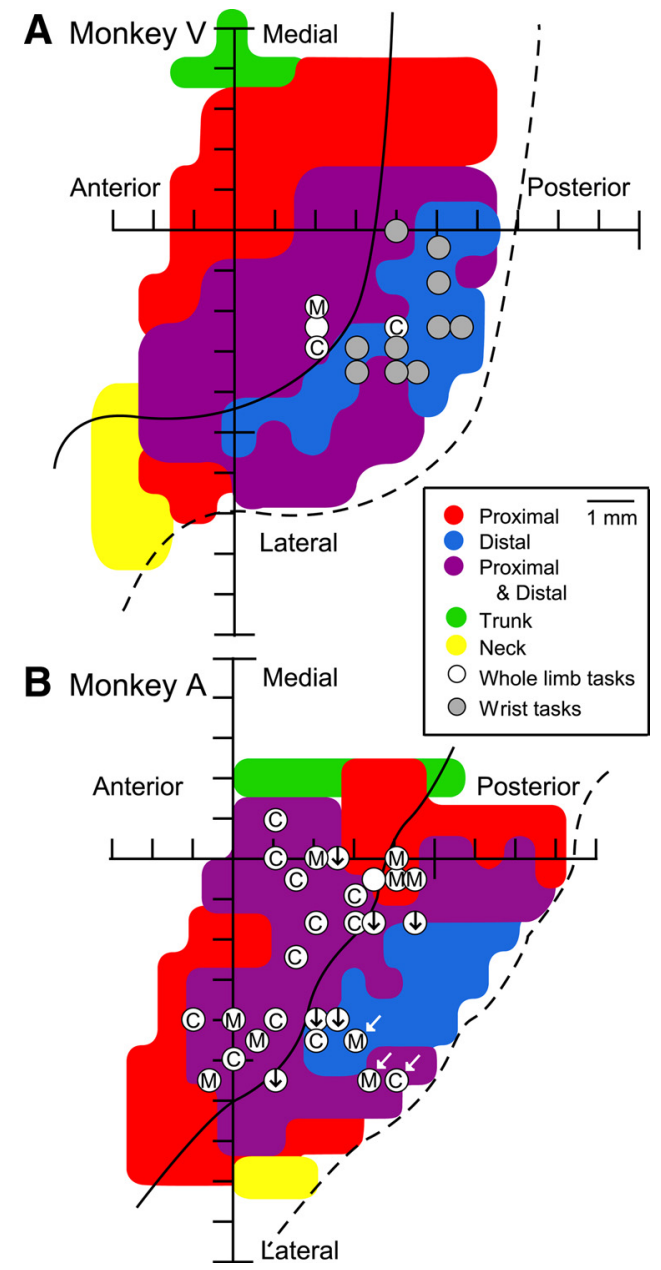

Figure 2. Cortical sites used to study EMG activity patterns associated with HFLD-ICMS of primary motor cortex in each of two monkeys $(V, A) . A, B$, Sites where HFLD-ICMS triggered EMG activity was collected with the reaching tasks (white circles) and the wrist tasks (gray circles) represented in two-dimensional coordinates after unfolding each monkey's precentral gyrus and corresponding muscle map based on effects obtained from StTA at $15 \mu \mathrm{A}$. Letters and symbols within the white circles denote hand end-point positions within the monkey's workspace produced with HFLD-ICMS at that site; midline (M), contralateral relative to the cortical site stimulated or ipsilateral relative to the affected arm $(C)$, and down from starting hand position (black arrows). Sites indicated by white arrows evoked movements to upper space near the monkey's head. Filled white circles with no letter or symbol are sites in which HFLD-ICMS did not produce a consistent hand end-point position.

Statistical data analysis. Effects of different starting hand position on HFLD-ICMS-evoked EMG responses were compared using the Student's $t$ test, $Z$ test, Mann-Whitney rank sum test, Pearson's correlation, and linear regression. In all tests, statistical significance was based on a $p \leq 0.05$.

\section{Results}

Data were obtained from stimulation of the left M1 cortex in two rhesus monkeys. HFLD-ICMS-evoked EMG activity was evaluated using stimulus intensities that produced consistent hand end-point positions ( 60 and $120 \mu \mathrm{A}$ ). A total of $42 \mathrm{M} 1$ sites were evaluated while the monkeys performed one of the tasks (Fig. 1). This included 14 sites in Monkey V (Fig. 2A) and 28 sites in Monkey A (Fig. $2 B$ ). Figure $2 A, B$ show the sites of HFLD-ICMS stimulation overlaid on muscle maps generated using StTA (individual biphasic stimulus pulses at $15 \mathrm{~Hz}$ and $15 \mu \mathrm{A}$ ). Sites where HFLD-ICMS-evoked EMG activity was obtained while the monkeys performed one of the reaching tasks (reach for peanuts or isometric push-pull) are marked with white dots and wrist tasks (isometric and concentric) are marked with gray dots. StTAs of EMG activity (15-120 $\mu \mathrm{A})$ were performed before each series of HFLD-ICMS experiments to confirm the intra-areal muscle representation and for comparison with HFLD-ICMS averages of EMG activity.

\section{Movements elicited with HFLD-ICMS}

For the reaching tasks, HFLD-ICMS was delivered when the monkey's hand reached the target position. This was just before the monkey grasped the food reward or as the monkey put the food reward into its mouth (hand position 4). Alternatively HFLD-ICMS was delivered while the monkey's hand was on the push-pull handle (Fig. 1A). Typically, 2-4 different hand starting positions were tested to maximize the change in joint angles. For example, to maximize the change in shoulder angle, hand positions 1 and 2 were tested. Alternatively, $\mathrm{A}$ and $\mathrm{D}$ or B and $\mathrm{C}$ were used. To maximize the change in elbow angle, starting hand positions 3 and 4 were tested. Joint angles associated with each starting hand position are given in Table 1.

HFLD-ICMS was applied to 32 cortical M1 sites while the monkeys performed the arm reaching tasks. Because these tasks relied heavily on proximal muscle activation, our cortical stimulation sites (29/32) emphasized the proximal and proximal-distal forelimb representations (Fig. 2). In $94 \%$ of sites tested (30/32), the arm movements drove the hand to converge toward a final common end-point regardless of starting hand position confirming the work of Graziano et al. (2002). HFLD-ICMS elicited arm movements resulted in the hand being brought toward the midline of the monkey at 10 sites, toward the contralateral (right) side at 14 sites and down from the starting position at six sites. Figure 2 gives the hand end-point locations relative to the cortical stimulation site. Although our sample of cortical sites is limited, we did not find any distinct clustering of hand end-point locations in the monkey most extensively studied using the whole limb reaching task (Monkey A; Fig. 2B). However, the three sites that produced movement of the hand to a level near the monkey's face (white arrows) were clustered deep in the central sulcus. HFLDICMS driven movement typically resulted in the hand being driven to the level of the waist or chest in front of the monkey or to sites contralateral to the hemisphere stimulated (ipsilateral to the moved arm). The six cortical sites which produced a downward movement of the monkey's hand from starting positions at the level of the monkey's head or chest produced somewhat more variable hand end-point positions. However, because the movements were consistently downward, we categorized them together (Fig. 2, downward pointing arrows). HFLD-ICMS at two cortical sites (Fig. 2, blank white circles) produced a mixture of these same movements without a consistent common final endpoint. HFLD-ICMS elicited movements at these sites often involved more than one joint. A broad range of shoulder and elbow movements were observed including elbow flexion and extension, shoulder adduction, extension and rotation.

HFLD-ICMS was applied to 10 sites while the monkey performed the wrist task (Fig. 2A). Some of these sites were also tested for whole arm movements with HFLD-ICMS. Although many of these sites did produce proximal arm movements, in addition to the wrist and digits, they typically did not yield highly consistent final hand end-points. This could be due to use of a relatively low stimulus intensity of $60 \mu \mathrm{A}$ (Van Acker et al., 2013). We therefore chose to focus on the wrist movements produced at these sites (Fig. 2A, gray filled circles). HFLD-ICMS produced wrist extension coupled with digit flexion (resulting in the hand taking on a fist like appearance) at these sites. 


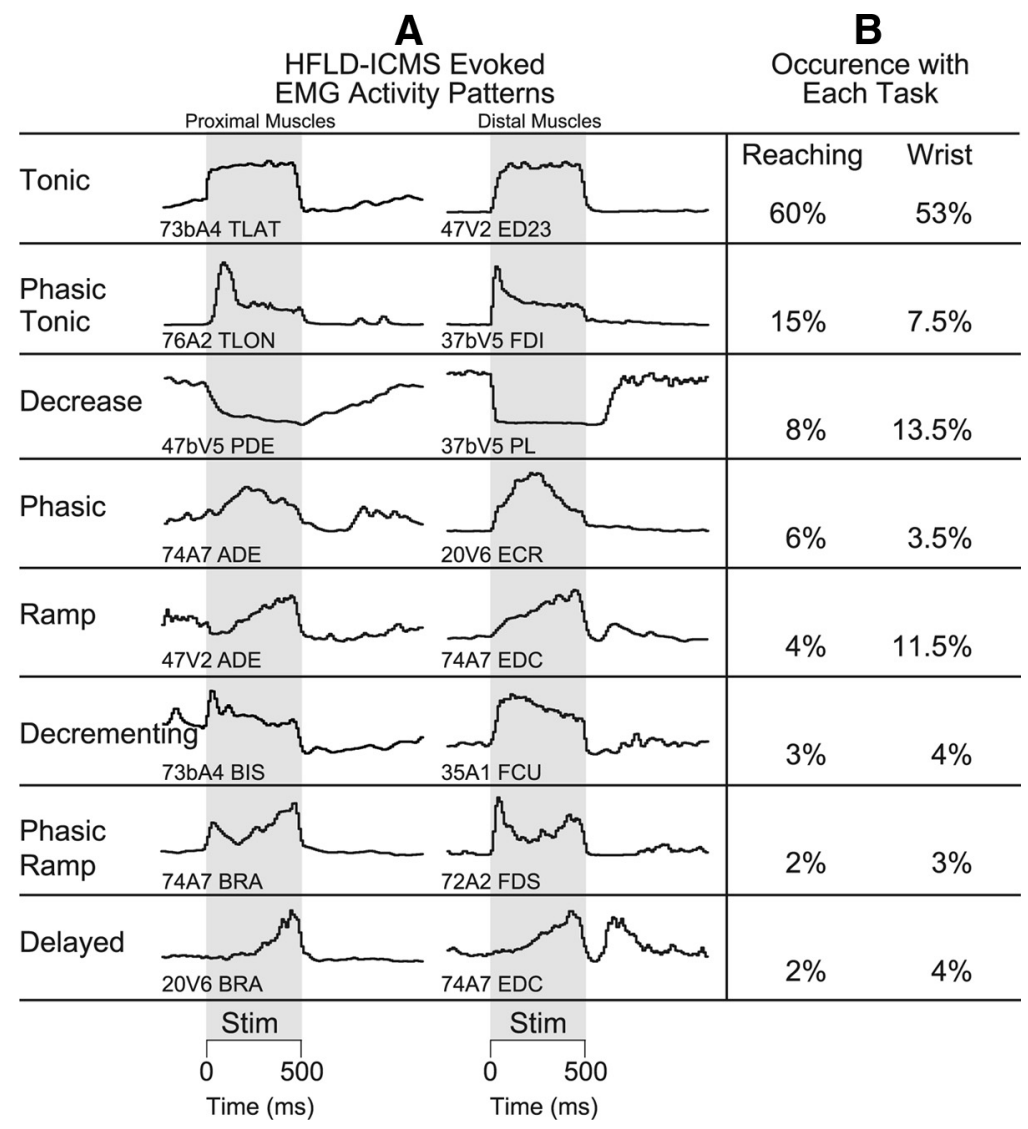

Figure 3. Qualitative characterization of EMG activation patterns evoked with HFLD-ICMS. A, Left column contains HFLD-ICMS evoked activity of proximal musculature. Right hand column contains HFLD-ICMS-evoked activity of distal musculature. $\boldsymbol{B}$, The prevalence of each activity pattern as a percentage of the total during performance of the reaching and wrist tasks respectively. Gray shading represents $500 \mathrm{~ms}$ stimulus train (100 pulses at 60 or $120 \mu \mathrm{A}$ ).

Temporal patterns of HFLD-ICMS-evoked EMG responses

A total of 768 cortical site-muscle pairs were investigated at two or more starting hand positions with the reaching tasks yielding 553 cortical site-muscle pairs with robust HFLD-ICMS-evoked EMG activity. HFLD-ICMS-evoked EMG activity of digit and intrinsic hand muscles were also investigated at different wrist positions with the wrist tasks. A total of 240 cortical site-muscle pairs were evaluated yielding 114 cortical site-distal muscle pairs with robust HFLD-ICMS-evoked EMG activity. The temporal patterns of EMG activity associated with the stimulus train were qualitatively evaluated for both the reaching and wrist tasks and placed into one of eight categories (Fig. 3). The EMG patterns and prevalence were similar for both movement tasks. The most common pattern (tonic: $58 \%$ of responses overall; $60 \%$ reaching task, $53 \%$ wrist task) was a sharp rise to a plateau which was essentially constant for the entire duration of the stimulus train. The second most common pattern (phasic-tonic: 14\% of responses overall; $15 \%$ reaching task, $7.5 \%$ wrist task) was an initial burst of activation followed by a decline to a plateau which was then constant for the duration of the stimulus. These two temporal activation patterns combined accounted for $72 \%$ of all responses observed. We characterized other activation patterns as phasic, ramp, decrementing, phasic-ramp, and delayed. HFLD-ICMS also evoked decreases in EMG activity relative to baseline. These responses consisted of sharp, immediate (relative to stimulus onset) declines in activity.

\section{HFLD-ICMS-evoked temporal EMG activation patterns are independent of hand position}

One way to assess HFLD-ICMS-evoked EMG activity dependence on starting hand position is to evaluate the stability of temporal EMG patterns (defined in Fig. 3) associated with individual muscles across each starting hand position tested. Eighty-three percent (1026/1242) of HFLD-ICMS-evoked temporal activation patterns remained the same for individual muscles across each starting hand position tested during the reach tasks and $63 \%(144 / 228)$ were the same for the two positions of the wrist task. When we evaluated temporal EMG activation patterns, but limited the data to the muscle group most affected by the joint angle change across different starting hand positions, we found similar high levels of independence. HFLDICMS-evoked temporal EMG activation patterns for shoulder muscles were independent of starting hand positions 1 and 2 (76\% remained the same). Similar results were obtained for the elbow muscles (72\%) across starting hand positions 3 and 4 , as well as with wrist/digit muscles (72\%).

Figure 4 shows one cortical site, where a large number of starting hand positions were tested using the reaching task. This cortical site was one in which HFLDICMS consistently drove the hand to an end-point near starting hand position $\mathrm{C}$ (Fig. 4B). A variety of temporal EMG activation patterns-evoked with HFLD-ICMS can be seen in these EMG records. We have color-coded each record by starting hand position and superimposed them for ease of comparison. Many of these muscles have virtually superimposable records whereas others show some variability. The most variable responses, however, are in muscles with relatively weak stimulus-evoked activation (e.g., ADE and PEC). Figure 4 further illustrates that in some cases the temporal activation patterns could be classified as different, yet, the actual evoked level of EMG activity was very similar. For example, stimulation at this cortical site drives BR activity down from a high prestimulus level of activity at starting hand position 1 and up from a low prestimulus level of activity at starting hand positions 2, 3, 4 (B and C). Although this muscle does show varying temporal patterns across starting hand positions (decreases in some cases and tonic increases in others), the stimulus clearly drives the muscle to a consistent level of activation in all cases. We previously noted this phenomenon of opposing responses depending on the starting level of EMG activity and attributed it to "neural hijacking" associated with high-frequency electrical stimulation (Griffin et al., 2011).

What other processes might be contributing to variability in temporal activation patterns in muscles at different hand starting positions? One possibility could be that as the limb is moving toward the final hand end-point position, afferent feedback is changing motoneuron excitability as well as cortical/subcortical excitability and this is reflected in the temporal profile of EMG 
activation. For example, if the excitability increases as the movement progresses it might be reflected as a ramp activation profile assuming other inputs remained constant. If the excitability decreases as the movement progresses, it would be reflected as a decrementing activation profile. A subset of our data are particularly relevant to this issue because HFLDICMS was applied during performance of an isometric wrist task (HFLD-ICMSevoked movement is prevented). Therefore, any dynamic movement related feedback was eliminated. The stability of the HFLD-ICMS-evoked distal muscle EMG pattern during the isometric wrist task improved by $6 \%$ after removing responses obtained during the concentric wrist task (72\% stability improved modestly to $78 \%$ ). This result suggests that feedback about dynamic movement can contribute to the variability seen in HFLD-ICMS temporal activation patterns.

Figure 5 shows results for HFLDICMS delivered to a single cortical site while the monkey performed the isometric wrist task. Although a high level of stability in temporal pattern of EMG activity associated with HFLD-ICMS is apparent in Figure 5, some variability was present. HFLD-ICMS was delivered at the beginning of the extension force-hold phase (column A) and at the beginning of the flexion force hold phase (column $B$ ). In each case (A and B), stimulation was applied with the wrist isometrically fixed in extension at $30^{\circ}$ or flexion at $30^{\circ}$ (black and gray records, respectively). The HFLD-ICMS-evoked EMG temporal profiles in each column are incredibly similar. Therefore, feedback about muscle length does not appear to be a large contributing factor for pattern variability.

Some pattern variability can be seen across columns. The two columns represent voluntary force production in opposite directions (toward extension in column A and toward flexion in column B). Much of the variability present can be attributed to the neural hijacking mechanism described previously (Griffin et al., 2011; Cheney et al., 2013) in which HFLD-ICMS eliminates natural input to corticospinal neurons and drives output to the same level regardless of natural input activity or conditions. For example, when the stimulus is applied during flexion force, FCR and PL activity decrease from a high prestimulus activity level. However, when the stimulus is applied during extension force, FCR and PL activity remains about the same throughout the stimulus train matching the level of activity driven by the stimulus during flexion force. FCU is yet another example of this phenomenon. Stimulation increased activity of FCU to a new tonic level when applied during extension force; however, when applied during flexion the activity is almost unchanged because it is already at the stimulus driven level. Overall, the pattern and magnitude of stimulus driven muscle activity were very consistent for individual muscles as well as for the summed EMG records (Fig. 5, bottom) confirming a high degree of consistency across all task conditions.

\section{The magnitude of HFLD-ICMS-evoked EMG activity is independent of hand position}

Stimulus driven EMG activation level provides another measure of the extent to which HFLD-ICMS-evoked responses are dependent on arm posture or joint angle. If the HFLD-ICMS-evoked EMG activity is independent of starting hand position, the magnitudes should be identical for different starting hand positions. Thus, plotting the mean evoked EMG level at one starting hand position against the mean evoked EMG level at a second starting hand position would yield a correlation coefficient of 1 and a regression line with a slope of 1. HFLD-ICMS evoked mean EMG activity across hand starting positions that produced the two most extreme joint angles for the shoulder (Fig. 6A), elbow (Fig. $6 B$ ), and wrist (Fig. 6C) were highly correlated $(r=0.95-0.96)$. In addition, the regression slopes associated with the HFLDICMS-evoked muscle activity levels were close to 1 (range, $0.96-$ 1.06). All confidence intervals included 1 (95\%).

Our magnitude analysis (Fig. 6A-C) included all the activated muscles at each cortical site stimulated. However, to test the natural circuit hypothesis we separated muscle groups (shoulder, elbow, and forearm) for comparison of effects elicited from starting hand positions that would produce the greatest changes in initial muscle length and possible changes in cortical/subcortical excitability. Once again, HFLD-ICMS-evoked mean muscle activity was highly correlated across starting hand positions. Further, stimulation did not yield a significant difference in activation level for either anterior or posterior shoulder muscles (Fig. 6D), elbow flexors and extensors (Fig. 6E), or forearm flexors and extensors (Fig. 6F). All regression slopes included 1 in their confidence intervals (95\%) except the elbow flexors. The elbow flexors show a significantly higher level of activation when 


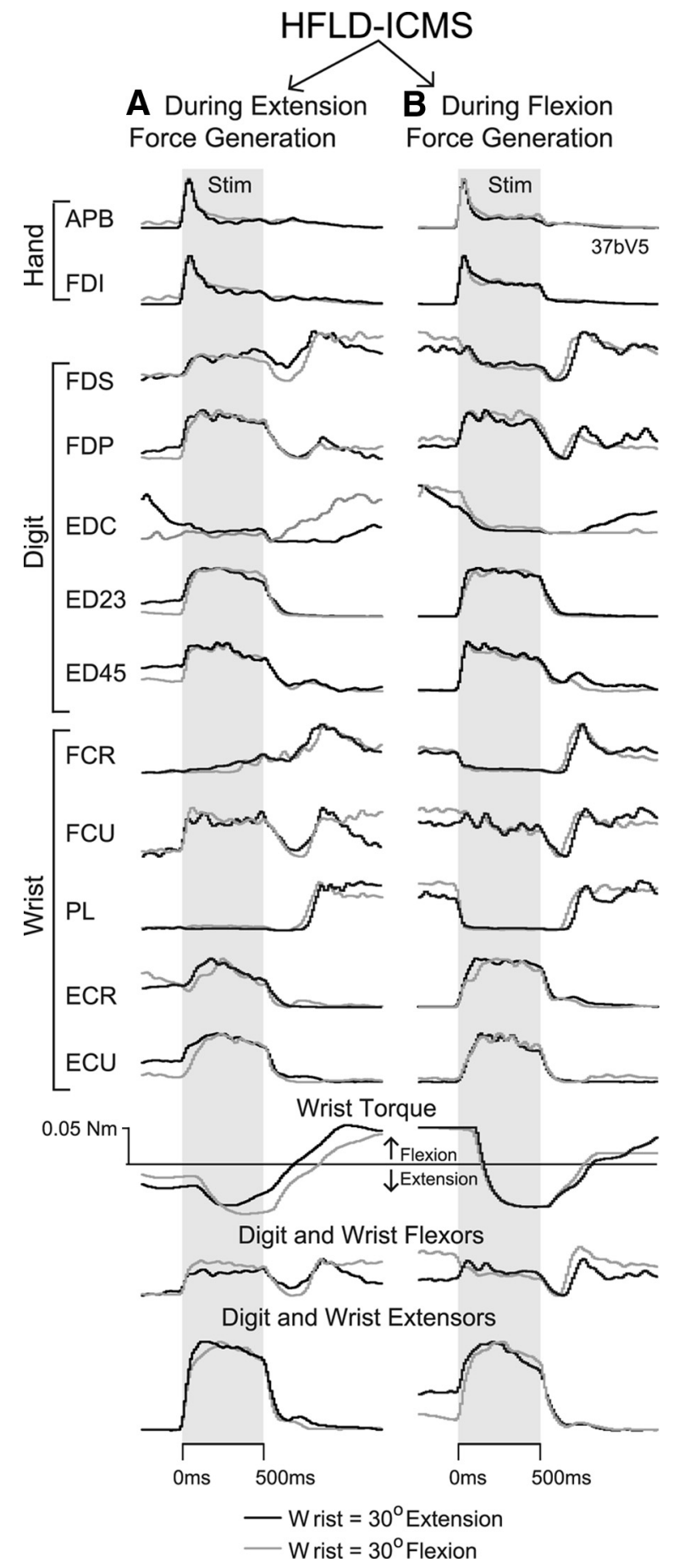

Figure 5. $\quad A, B$, Forearm EMG activation patterns associated with HFLD-ICMS delivered during $(\boldsymbol{A})$ generation of voluntary extension force, and $(\boldsymbol{B})$ generation of voluntary flexion force. HFLD-ICMS was applied at this cortical site with the wrist task manipulandum locked in place at $30^{\circ}$ of wrist extension (black traces) or $30^{\circ}$ of flexion (gray traces). Gray shading represents the 500 ms stimulus train (100 pulses at $120 \mu \mathrm{A})$. Stimulus-evoked EMG activity for the same muscles are displayed at the same gain. With the wrist free to move, HFLD-ICMS at this site drove the wrist toward extension.

the elbow was flexed and lower activation when the elbow was extended although the difference was small.

\section{HFLD-ICMS responses at hand positions that straddle the end-point position}

One of the primary objectives of this study was to choose between two possible mechanisms (activation of a natural output circuit versus stimulus driven equilibrium) that might account for the observation that HFLD-ICMS at individual cortical sites produces movement to common end-point positions regardless of the starting hand position. The natural circuit activation hypothesis predicts a reciprocal activation pattern of agonist and antagonist muscles depending on the starting hand position replicating what occurs in natural voluntary movement. The stimulus driven equilibrium hypothesis predicts a constant pattern of agonist and antagonist muscle activation regardless of starting hand position. The most stringent test of this hypothesis is provided by data from starting hand positions on opposite sides of the end-point position. We have identified a subset of our data that is directly relevant and matches these criteria. At 10 of our cortical sites, HFLD-ICMS drove the hand to the midline of the monkey's body (in front of the face or abdomen). Starting hand positions 1 and 2 straddle these end-points and therefore allow an opportunity to test the hypotheses described above. Since movement starting from the left or right and ending at the midline relies heavily on shoulder muscle activation, our analysis emphasized the shoulder muscles. Analysis was limited to 38 cortical site-shoulder muscle pairs, with robust HFLD-ICMS-evoked EMG activity, tested with starting hand positions 1 and 2 .

For shoulder muscle activation to agree with the natural circuit hypothesis, posterior shoulder muscles (TMAJ, LAT, and PDE) should show increased activity when the hand is at starting position 1 and decreased activity when the hand is at starting position 2. Anterior shoulder muscles (ADE and PEC) should show the opposite activation (decreased activation when the hand is at starting position 1 and increased activation at starting hand position 2). Our results support the stimulus driven equilibrium hypothesis. The level of HFLD-ICMS-evoked shoulder muscle EMG activity at these sites is stable and independent of starting hand position. HFLD-ICMS-evoked shoulder muscle activity levels were highly correlated across the two starting hand positions $(r=0.85)$. This high level of correlation improved or stayed the same when we evaluated anterior shoulder muscles $(r=0.96)$ and posterior shoulder muscles $(r=0.85)$ separately. The regression slopes associated with the HFLD-ICMS-evoked anterior shoulder (1.01) and posterior shoulder (1.21) muscle levels were not significantly different from 1 (95\% confidence interval).

\section{HFLD-ICMS-evoked onset latency}

The onset latency of muscle activation provides additional insight concerning the mechanism underlying HFLD-ICMS-evoked EMG activity. Onset latencies were measured as the point where the record left the pretrigger baseline toward a peak. All HFLDICMS-evoked muscle activation patterns were evaluated and separated into the same two categories: (1) muscles with facilitation effects present in both HFLD-ICMS triggered averages of EMG activity and in low intensity (15 $\mu \mathrm{A})$ StTAs of EMG activity, and (2) those with effects present in HFLD-ICMS with no effects in StTAs. Early onset latency HFLD-ICMS effects $(<20 \mathrm{~ms})$ were present in almost twice as many ( $82 \%$; mean, $10.9 \mathrm{~ms}$ ) cases when PStF was present in StTAs as compared with when PStF was absent (47\%; mean, $11.9 \mathrm{~ms})$. The mean onset latency for all HFLD-ICMS effects with PStF present in StTAs was $20 \mathrm{~ms}$ earlier than HFLD-ICMS effects where PStF in StTAs was absent (mean, $18.7 \mathrm{vs} 38.8 \mathrm{~ms}$ ). Very late onset latencies ( $>100 \mathrm{~ms}$ ) were present in a larger percentage of records ( $8 \%$; mean, $165.2 \mathrm{~ms}$ ) when PStF in StTAs was absent compared to when it was present (2\%; mean, $132 \mathrm{~ms}$ ). These results show that cortical site-muscle pairs with poststimulus effects present in low intensity StTA also show ro- 
bust short latency activation of the same muscles with HFLD-ICMS. This most likely reflects muscle activation through a relatively direct route to motoneurons. The longer latency HFLD-ICMS effects at sites lacking effects in StTAs suggest activation of muscles through a polysynaptic route to motoneurons.

\section{Discussion}

Our data support the finding that HFLDICMS at a particular cortical site produces movements of the forelimb toward a final common end-point position independent of the starting position (Graziano et al., 2002; Graziano et al., 2005). We also found that muscle activation associated with HFLD-ICMS is largely stable in sign, magnitude, and temporal pattern independent of starting position or arm posture. This result is consistent with previous findings with HFLD-ICMS (Griffin et al., 2011) and StTA (Griffin et al., 2009). Our data are most consistent with the view that HFLD-ICMS produces activation of agonist and antagonist muscles, most commonly as a sustained tonic or phasic-tonic pattern of activity. The activated muscles then generate torque and produce movements according to their length-tension properties until an equilibrium position is achieved. At each joint, a specific level of activation of agonist and antagonist muscles will correspond to a specific joint angle. The fact that the magnitude and pattern of activation of muscles is the same regardless of starting position of the limb means that the limb will always seek and find the same common end-point where an equilibrium of muscle torques is achieved at each joint. Our results are not consistent with the view that muscle activation associated with HFLD-ICMS varies systematically as a function of arm position or joint angle. Further, our results do not replicate the EMG patterns that would be associated with natural, active movements over the same trajectory as the stimulus evoked movements.

\section{HFLD-ICMS-evoked muscle activation is independent of arm posture/joint angle}

Due to the high-frequency and long duration of the ICMS used in this study, it is likely that excitation spread to premotor, corticoreticulospinal, and corticorubrospinal pathways. As a result, these pathways are also likely to have contributed to the output effects on muscles we observed. Physiological spread from ICMS was emphasized most recently by Maier et al. (2013) who showed that even single-pulse ICMS elicits robust cortical I waves and transsynaptic activation of cortical cells relatively distant from the site of stimulation. One mechanism to explain HFLD-ICMSevoked movements is that the stimulus replaces the naturally positions indicated were tested.

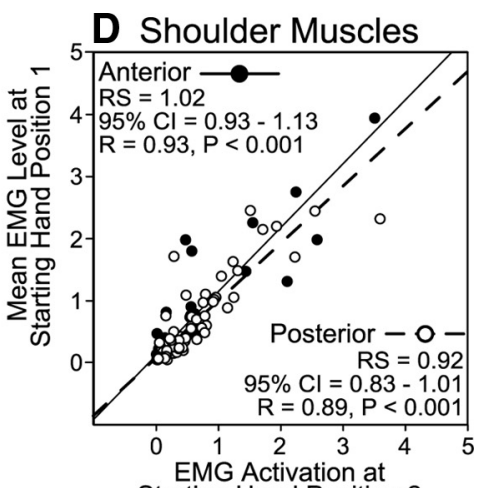

Starting Hand Position 2

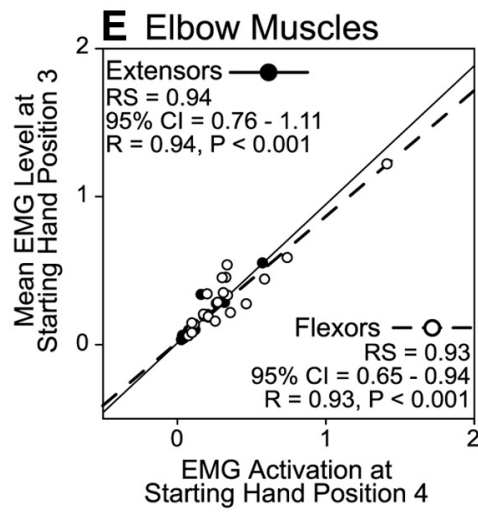

F Wrist \& Digit Muscles

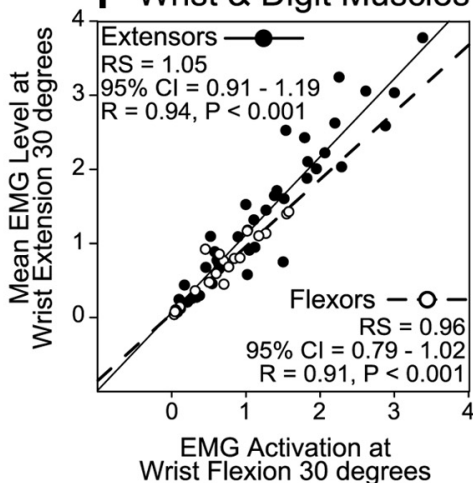

Wrist Flexion 30 degrees

Figure 6. $\boldsymbol{A}-\boldsymbol{C}$, Relationship between the magnitudes of HFLD-ICMS-evoked EMG activity at starting hand positions that produced the most extreme positions of the shoulder $(\boldsymbol{A})$, elbow $(\boldsymbol{B})$, and wrist $(\boldsymbol{C})$. Linear regression lines are plotted, 95\% Starting hand positions relate to Figure 1.D-F, The muscles are separated by joint and stability of magnitude is tested for the hand positions that produce the largest changes in angle for the muscles acting at that joint. Agonist and antagonist muscles are plotted separately. Each data point represents a cortical site-muscle pairs for all cases in which the starting hand

occurring neuronal activity locally within the motor cortex, and remotely in premotor and subcortical motor circuits with a new stimulus driven level of output. This stimulus driven output essentially hijacks the corticospinal circuitry and replaces natural input to motoneurons with a new, stimulus driven level of input (Griffin et al., 2011; Cheney et al., 2013). This mechanism would predict a relatively stable pattern of muscle activation independent of changes in limb posture or joint position. We found that HFLD-ICMS-evoked EMG activation producing hand movements to a final common end-point to be highly stable with regard to the magnitude of activation (Fig. 6) and the temporal pattern of activation (Figs. 4, 5). HFLD-ICMS at a particular site in $\mathrm{M} 1$ consistently activates the same set of muscles regardless of the starting or ending hand position. HFLD-ICMS did not acti- 
vate a set of agonist muscles for movements in one direction and antagonists for movements in the opposite direction as occurs during natural movements.

One strikingly consistent finding was that varying starting hand position did not change the magnitude of HFLD-ICMSevoked muscle activity. Magnitudes of the HFLD-ICMS elicited effects were well correlated at the different hand starting positions that produced the largest joint angle changes. Moreover, the linear regressions had slopes that were not significantly different from one ( $95 \%$ confidence intervals). There were a small number of cases in which starting hand position did have an impact on HFLD-ICMS-evoked EMG activity (Fig. 6E, elbow flexor muscles). Overall, the data demonstrate that at any single site in $\mathrm{M} 1$, HFLD-ICMS activated muscles to the same level independent of the starting or ending hand position. In other words, HFLDICMS did not produce stronger activation of agonist muscles for movements in one direction and weaker activation or suppression of the same muscles for movements in the opposite direction. Previous studies (Graziano et al., 2002; Graziano et al., 2004) have reported changes in output effects as a function of elbow joint angle. These studies involved recording HFLD-ICMS elicited activity from biceps and triceps at different elbow angles. They reported that stimulus elicited effects in biceps became stronger and triceps weaker as the elbow was extended. Conversely, as the elbow was flexed, the stimulus elicited effects in triceps strengthened and biceps weakened. One potentially important difference between our approach and that of Graziano et al. (2002, 2004) is that our monkeys were not anesthetized or tranquilized with ketamine. It is feasible that in the absence of voluntary movement and related modulation of spinal cord inputs, motoneuron excitability might become more heavily dominated by spindle afferent input associated with joint angle changes. The changes reported by Graziano et al. (2004) are consistent with expected changes in motoneuron excitability associated with changes in muscle spindle afferent input at different joint angles.

\section{Mechanism of HFLD-ICMS-evoked movements}

Our results are consistent with the neural hijacking hypothesis described above (Griffin et al., 2011; Cheney et al., 2013). Descending stimulus driven input to motoneurons dominates EMG activity, elicits coactivation of multiple forearm muscles, which produce active forces at joints resulting in movement of the arm. HFLD-ICMS output effects obtained at individual sites in M1 were consistent across different starting hand positions, with reference to both the set of muscles activated and the activation levels of those muscles. We propose that the limb achieves its final position largely determined by the balance of activity evoked in agonist and antagonist muscles. It is well known that the amount of force a muscle generates is dependent on its length (Gordon et al., 1966; Rack and Westbury, 1969) and level of activation (Rothwell, 1994). Length-tension curves have been used to describe the change in muscle length, plotted as change in joint angle, given the level of activation (tension) associated with two antagonist prime mover muscles. The intersection of two length-tension curves represents the joint angle at which the torque produced by flexors equals the opposing torque produced by extensors - the equilibrium position (Rothwell, 1994). Because muscle tension is known to be linearly related to the mean level of EMG activity (Milner-Brown and Stein, 1975), the slopes of the length-tension curves can be shifted upward or downward depending on the level of muscle activation.

For example, Figure 7 illustrates, in a simplified fashion, how the length-tension relationships of biceps and triceps ( $y$-axes)

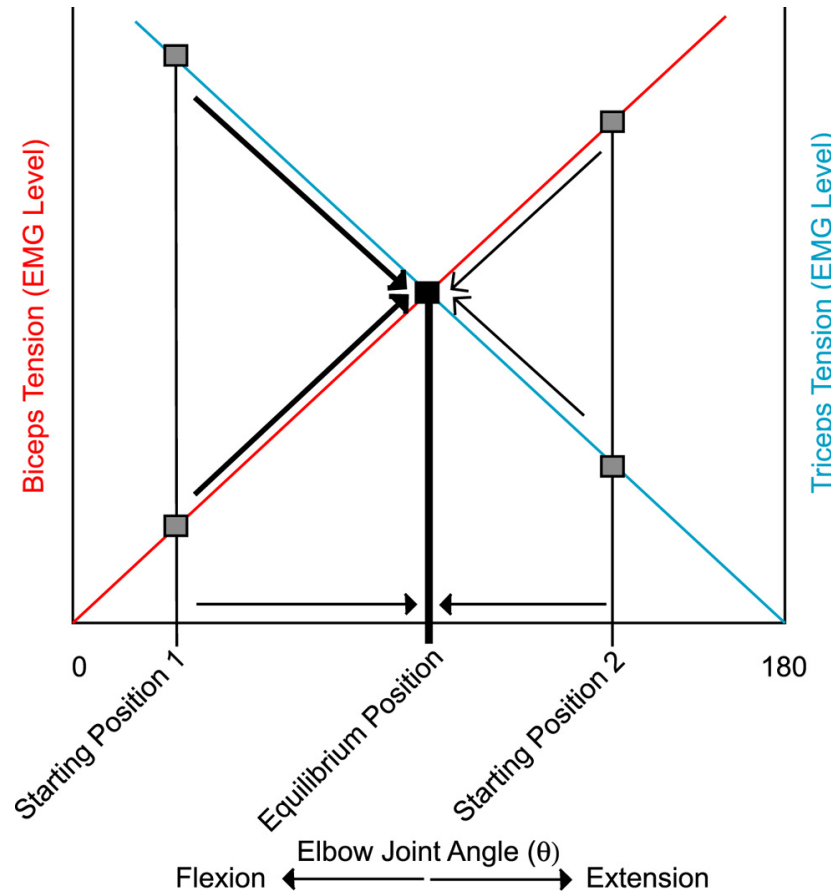

Figure 7. Length-tension relationships of biceps and triceps specify elbow joint angle. Muscle tension is plotted along the $y$-axis against elbow joint angle $(\theta)$ as opposed to muscle length on the $x$-axis. The red line represents the length-tension curve of biceps and the blue line triceps at given muscle activity levels. Gray squares represent levels of muscle tension produced by biceps and triceps at the beginning of two example starting hand positions. Given this level of HFLD-ICMS-evoked biceps and triceps activity, these muscles will produce movement from either starting hand position to a final hand position reflecting the equilibrium point (heavy and light diagonal arrows). The black square represents the point of intersection between lengthtension curves; the equilibrium position of the joint (heavy vertical line). Horizontal lines represent corresponding changes in elbow angle associated with movement toward the equilibrium position.

might interact to specify elbow joint angle ( $x$-axis). The red line represents the length-tension curve of biceps and the blue line triceps at a particular level of muscle activity. The gray squares represent two different levels of muscle tension that would be produced by biceps and triceps at two starting hand positions, one of which is a flexed elbow angle (starting position 1) and the other a more extended elbow angle (starting position 2). Given this level of HFLD-ICMS-evoked biceps and triceps activity, these muscles will produce movement from any starting hand position to a final hand position reflecting the equilibrium position represented by the black square. For example, with starting hand position 1 (Fig. $1 A a$, position 4 ), triceps tension is greater than biceps. As a result, triceps will shorten along its lengthtension curve as biceps lengthens (indicated by heavy arrows). As triceps shortens, the amount of force production decreases, while as biceps lengthens its force production will increase. Eventually, the forces produced by both muscles reach equilibrium and the movement stops. With starting hand position 2 (Fig. $1 \mathrm{Aa}$, position 3), biceps tension starts out greater than triceps. Biceps shortens along its length-tension curve as triceps lengthens (indicated by light arrows) until the forces reach equilibrium and movement stops. As illustrated in this figure, the equilibrium position will always be the same, regardless of starting position, if the level of EMG activation in each muscle is the same. Different levels of muscle activity produce different sets of length-tension curves, resulting in movement to different final end-point positions reflecting the equilibrium position between those forces. 
Our data show that, in most cases, HFLD-ICMS produces the same level of muscle activity independent of starting hand position. These levels of agonist and antagonist muscle activity produce corresponding joint forces resulting in movement according to the length-tension properties of the muscles to a specific equilibrium point (joint position) that will be the same for the same level of muscle activity. This equilibrium point will be achieved regardless of the initial length of the agonist and antagonist muscles, that is, regardless of the starting position of the limb. The final end-point position of the hand represents the equilibrium position of forces acting at the forelimb joints due to all the muscles activated from stimulation at a given cortical site. Finally, it should be noted that the pattern of HFLD-ICMSevoked EMG activation and underlying mechanism is fundamentally different from the mechanism underlying the patterns of EMG activation associated with natural voluntary movement.

\section{References}

Aflalo TN, Graziano MS (2006a) Partial tuning of motor cortex neurons to final posture in a free-moving paradigm. Proc Natl Acad Sci U S A 103: 2909-2914. CrossRef Medline

Aflalo TN, Graziano MS (2006b) Possible origins of the complex topographic organization of motor cortex: reduction of a multidimensional space onto a two-dimensional array. J Neurosci 26:6288-6297. CrossRef Medline

Brown SH, Cooke JD (1990) Movement-related phasic muscle activation: I. Relations with temporal profile of movement. J Neurophysiol 63:455464. Medline

Buys EJ, Lemon RN, Mantel GW, Muir RB (1986) Selective facilitation of different hand muscles by single corticospinal neurones in the conscious monkey. J Physiol 381:529-549. Medline

Cheney PD, Fetz EE (1980) Functional classes of primate corticomotoneuronal cells and their relation to active force. J Neurophysiol 44:773-791. Medline

Cheney PD, Griffin DM, Van Acker GM 3rd (2013) Neural hijacking: action of high-frequency electrical stimulation on cortical circuits. Neuroscientist 19:434-441. CrossRef Medline

Gordon AM, Huxley AF, Julian FJ (1966) The variation in isometric tension with sarcomere length in vertebrate muscle fibres. J Physiol 184:170-192. Medline

Graziano MS, Taylor CS, Moore T (2002) Complex movements evoked by microstimulation of precentral cortex. Neuron 34:841-851. CrossRef Medline
Graziano MS, Patel KT, Taylor CS (2004) Mapping from motor cortex to biceps and triceps altered by elbow angle. J Neurophysiol 92:395-407. CrossRef Medline

Graziano MS, Aflalo TN, Cooke DF (2005) Arm movements evoked by electrical stimulation in the motor cortex of monkeys. J Neurophysiol 94: 4209-4223. CrossRef Medline

Griffin DM, Hudson HM, Belhaj-Saïf A, Cheney PD (2009) Stability of output effects from motor cortex to forelimb muscles in primates. J Neurosci 29:1915-1927. CrossRef Medline

Griffin DM, Hudson HM, Belhaj-Saif A, Cheney PD (2011) Hijacking cortical motor output with repetitive microstimulation. J Neurosci 31: 13088-13096. CrossRef Medline

Hoffman DS, Strick PL (1999) Step-tracking movements of the wrist: IV. Muscle activity associated with movements in different directions. J Neurophysiol 81:319-333. Medline

Maier MA, Kirkwood PA, Brochier T, Lemon RN (2013) Responses of single corticospinal neurons to intracortical stimulation of primary motor and premotor cortex in the anesthetized macaque monkey. J Neurophysiol 109:2982-2998. CrossRef Medline

McKiernan BJ, Marcario JK, Karrer JH, Cheney PD (1998) Corticomotoneuronal postspike effects in shoulder, elbow, wrist, digit, and intrinsic hand muscles during a reach and prehension task. J Neurophysiol 80: 1961-1980. Medline

Milner-Brown HS, Stein RB (1975) The relation between the surface electromyogram and muscular force. J Physiol 246:549-569. Medline

Park MC, Belhaj-Saif A, Cheney PD (2000) Chronic recording of EMG activity from large numbers of forelimb muscles in awake macaque monkeys. J Neurosci Methods 96:153-160. CrossRef Medline

Park MC, Belhaj-Saïf A, Gordon M, Cheney PD (2001) Consistent features in the forelimb representation of primary motor cortex in rhesus macaques. J Neurosci 21:2784-2792. Medline

Rack PM, Westbury DR (1969) The effects of length and stimulus rate on tension in the isometric cat soleus muscle. J Physiol 204:443-460. Medline

Rothwell J (1994) Control of human voluntary movement, Ed 2. London: Chapman and Hall.

Stoney SD Jr, Thompson WD, Asanuma H (1968) Excitation of pyramidal tract cells by intracortical microstimulation: effective extent of stimulating current. J Neurophysiol 31:659-669. Medline

Van Acker GM 3rd, Amundsen SL, Messamore WG, Zhang HY, Luchies CW, Kovac A, Cheney PD (2013) Effective intracortical microstimulation parameters applied to primary motor cortex for evoking forelimb movements to stable spatial end points. J Neurophysiol 110:1180-1189. CrossRef Medline 